\title{
Schisandra chinensis inhibiting TGFß-induced activation of hepatic stellate cells
}

\author{
Soon Young Shin ${ }^{1} \cdot$ Junho Lee $^{3} \cdot$ Ha-Na Gil ${ }^{1} \cdot$ You Jung Jung ${ }^{1} \cdot$ \\ Gyeong Lan Kim² - Gil Hak Kang ${ }^{2}$ Yoongho Lim $^{3}$
}

Received: 17 July 2018/ Accepted: 6 September 2018/Published online: 18 September 2018

(C) The Korean Society for Applied Biological Chemistry 2018

\begin{abstract}
Hepatic fibrosis is one of the critical steps contained in the pathogenesis of liver cirrhosis. Excessive deposition of collagen contributes to the development of fibrosis in chronic liver injury. Activation of hepatic stellate cells (HSCs) plays an important role in fibrogenesis and is accountable for providing extracellular matrix components. The berry of Schisandra chinensis has been known to exert hepatoprotective properties. However, its effect on HSCs is not completely understood. Therefore, in this study, we investigated the inhibitory effect of its ethanolic extract (SBE) on hepatic fibrogenesis. We found that SBE treatment effectively reduced the serum levels of alanine aminotransferase and aspartate aminotransferase as well as collagen deposition in the hepatic parenchyma in a thioacetamide-induced hepatic fibrosis mouse model. Moreover, SBE inhibited transforming growth factor $\beta$ (TGF $\beta$ )-induced mRNA expression of $\alpha$-smooth muscle actin ( $\alpha$ SMA) and collagen type $1 \alpha 1$ (COL1A1) in HSCs, suggesting that SBE exerts anti-fibrotic activity by attenuating TGF $\beta$-induced HSC activation. To identify the active components of SBE accountable for HSC inhibition, SBE was further partitioned based on the hydrophobicity of the solvents such as water, $n$-butanol, ethyl acetate, chloroform, and $n$-hexane. The $n$-hexane fraction was selected
\end{abstract}

Yoongho Lim

yoongho@konkuk.ac.kr; yoongholim@gmail.com

1 Department of Biological Sciences, Cancer and Metabolism Institute, Konkuk University, Seoul 05029, Republic of Korea

2 Mungyeong Miso Co. Ltd., Mungyeong, Gyeongbuk 36932, Republic of Korea

3 Division of Bioscience and Biotechnology, BBRC, Konkuk University, Seoul 05029, Republic of Korea and further separated using analytical high-performance liquid chromatography. We found that six lignans contained in the $n$-hexane fraction strongly reduced TGF $\beta$ induced expression of both $\alpha \mathrm{SMA}$ and COL1A1 mRNA. These data suggest that at least six lignans contained in SBE have the strong potential to prevent TGF $\beta$-induced HSC activation.

Keywords Alpha-smooth muscle actin · Collagen type 1 $\alpha 1$. Hepatic fibrosis - Hepatic stellate cells - Schisandra . Transforming growth factor $\beta$

\section{Introduction}

The liver is the largest single organ in our body, is widely distributed over the right upper abdomen and left upper abdomen, and is known to be the most commonly damaged organ in both blunt and penetrating injuries. The liver is largely divided into the left liver and the right liver and is divided into 8 regions according to the running of the veins, arteries, and bile ducts. The liver is divided into the arterial and portal vein. The blood vessels are very abundant in the receiving organs, and a large amount of bleeding may occur when they are damaged. In addition, secondary damage of the bile ducts in the liver may be accompanied by stomach, duodenum, pancreas, and adrenal glands surrounding the liver. The liver is an important organ that plays a pivotal role in the detoxification of metabolism and various endogenous and exogenous harmful substances. However, hepatocytes are destroyed, liver function is less than half, and special symptoms do not appear. Because many drugs are metabolized through the liver, if we take the drug over a liver function, we may 
develop a herd that can cause liver dysfunction. Hepatocyte damage refers to necrosis of hepatocytes, fatty hepatitis or cognitive vaginosis. It can be caused by various drugs including tetracycline, ritonavir, risperidone, phenytoin, valproate, nevirapine, ketoconazole, pyrazinamide, rifampin, trazodone, halothane, aspirin, diclofenac, and acetaminophen. If hepatocyte damage persists for a long time, hepatic fibrosis accumulates in the liver. Hepatic fibrosis is an inflammatory response to liver injury, characterized by excessive accumulation of extracellular matrix (ECM) proteins in the hepatic parenchyma [1]. When hepatocytes are damaged by various stimuli, such as inflammation, alcohol poisoning, or toxic drugs, infiltrating inflammatory lymphocytes and resident liver macrophages (Kupffer cells) release various growth factors, including transforming growth factor- $\beta$ (TGF $\beta$ ), platelet-derived growth factor, and tumor necrosis factor- $\alpha$. Hepatic stellate cells (HSCs), also known as Ito cells or lipocytes, are star-like-shaped cells located between hepatocytes and sinusoidal endothelial cells. HSCs represent 5-8\% of all liver cells and play a role in the uptake and storage of vitamin A [2]. HSCs are activated by these growth factors and play a critical role in promoting tissue regeneration by producing collagen-rich ECM proteins [3]. However, when hepatocyte destruction and regeneration occur repeatedly, massively accumulated collagens are transformed from fine fibrils to thick fibers, leading to the development of hepatic fibrosis [4]. Advanced hepatic fibrosis disrupts the hepatic architecture and eventually causes liver cirrhosis and dysfunction [1]. As fibrosis is a reversible reaction [5], it has been suggested that the prevention or treatment of hepatic fibrosis can be achieved by blocking the synthesis of collagen fibers. Therefore, it is important to inhibit the activity of HSCs to prevent the progression of hepatic fibrosis [3]. Unfortunately, there is no good way to treat hepatic fibrosis.

Schisandra chinensis is a medicinal herb that belongs to the Schisandraceae family, whose growth range extends from northeast China, Korea, and Japan to the most eastern part of Russia [6, 7]. Because it tastes sour, sweet, spicy, bitter, and salty, it is called omiza in Korea. The berry of $S$. chinensis is widely used in traditional Chinese, Japanese, Russian, and Korean medicine [7]. One of Korean traditional medical books, Donguibogam reports the berry of $S$. chinensis can restore fatigue, strengthen heart function, improve blood circulation, and prevent memory loss or slowing of thinking ability. The berry of $S$. chinensis contains various active compounds and exerts a wide range of biological activities, including antioxidant, anti-inflammatory, antimicrobial, anti-allergic, anti-tumor, anti-angiogenic, antiseptic, and anti-hepatotoxic effects, and protective effects against cardiovascular disease and on pancreatic $\beta$-cells in diabetic rats and on oxidative stress and cytotoxic activities. [7]. Many studies have reported that dibenzocyclooctadiene lignans, such as schisandrin A (deoxyschisandrin), schisandrin B (gomicin N), gomisin A (schisandrol B), and gomisin $\mathrm{J}$, have protective effects against liver injury [8-12]. In addition, more lignans including schisandrol A, gomisin $\mathrm{D}$, micrantherin $\mathrm{A}$, pregomisin, angeloylgomisin $\mathrm{H}$, benzoylgomisin $\mathrm{H}$, angeloylgomisin $\mathrm{Q}$, gomisin $\mathrm{G}$, gomisin $\mathrm{F}$, gomisin $\mathrm{K}$, gomisin $\mathrm{K} 2$, schisantherin $\mathrm{A}$, tigloylgomisin $\mathrm{P}$, schisanhenol, gomisin L1, schisantherin C, gomisin M1, gomisin $\mathrm{M} 2$, epigomisin $\mathrm{O}, \gamma$-schisandrin, benzoylisogomisin $\mathrm{O}$, angeloylisogomisin $\mathrm{O}$, schisandrin $\mathrm{C}$, and angeloylgomisin $\mathrm{O}$, and polyphenols including 5-O-caffeoylquinic acid, quercetin-3-O-rutinoside, quercetin-3-O-glucoside, quercetin, kaempferol, 3-O-caffeoylquinic acid, 4-O-caffeoylquinic acid, 4-O-p-coumaroylquinic acid, 3-O-pcoumaroylquinic acid, trans- and cis-5-O-p-coumaroylquinic acid, quercetin-3-O-glucoside-7-O-rhamnoside, quercetin-3-O-galactoside, quercetin-3-O-xyloside, kaempferol-3-O-glucoside-7-O-rhamnoside, kaempferol-3O-glucoside, kaempferol-3-O-arabinose, 6"-O-(4-hydroxycinnamoyl)-astragalin have been identified as the components of S. chinensis.

However, the effect of the berry of S. chinensis on inhibiting HSCs has not been well characterized. In this study, we aimed to determine the anti-HSC effect of the ethanolic extracts of the berry of $S$. chinensis (named as SBE) against TGF $\beta$-stimulated HSCs and identify the bioactive ingredient in SBE responsible for inhibiting TGF $\beta$-induced $\alpha$-smooth muscle actin ( $\alpha$ SMA) and collagen type $1 \alpha 1$ (COL1A1) expression.

\section{Materials and methods}

\section{Chemicals and reagents}

Thioacetamide was obtained from Sigma-Aldrich (St. Louis, MO, USA). TGF $\beta$ was purchased from R\&D Systems (Minneapolis, MN, USA). Lignans including gomisin $\mathrm{D}$, gomisin $\mathrm{G}$, gomisin $\mathrm{H}$, gomisin $\mathrm{J}$, gomisin $\mathrm{N}$, gomisin $\mathrm{O}$, angeloylgomisin $\mathrm{H}$, angeloylgomisin $\mathrm{O}$, and benzoylgomisin $\mathrm{O}$ were purchased from Biopurify (Chengdu, Sichuan, China). Other chemicals were purchased from Sigma-Aldrich.

\section{Preparation of Schisandra extracts}

The berry of $S$. chinensis was collected from Mungyeong Miso farm (Gyeongbuk, Korea) in September 2016, and a voucher herbarium specimen was deposited at Konkuk University, Korea. The berry was dried under a shaded lot, and the dried berry $(9000 \mathrm{~g})$ was extracted three times with ethanol for three days. The ethanolic extract $(2747.51 \mathrm{~g}$, 
$30.53 \%$ ) was subjected to sequential separation with solvents of different polarities. Five fractions were obtained: $n$-hexane $(3.35 \%)$, chloroform $(0.67 \%)$, ethyl acetate $(11.12 \%), n$-butanol $(59.37 \%)$, and aqueous fraction $(22.99 \%)$. Whole fractions were freeze-dried and stored at $20{ }^{\circ} \mathrm{C}$ until further experiments. Analytical high-performance liquid chromatography (HPLC) was performed to analyze the components in SBE. The method of the analytical HPLC for the identification of the components followed the procedure provided by the manufacture.

\section{Induction of hepatic fibrosis with thioacetamide in Balb/c mice}

Balb/c mice were purchased from Youngbio (Seongnam, Gyeonggi-do, Korea). Mice were divided into 3 groups ( $n=3$ /group): control, TAA only, and TAA with SBE (Fig. 1A). Control group mice were intraperitoneally administered phosphate-buffered saline (PBS) solution. Hepatic fibrosis was induced by intraperitoneal injection of TAA $(100 \mathrm{mg} / \mathrm{kg})$ three times per week for a total of 8 weeks. SBE (300 mg/kg) was orally administered once per day for 10 days before TAA treatment, and then TAA and SBE were administered on the same schedule (Fig. 1A). After 8 weeks of TAA treatment, liver and blood samples were collected. Animal experiments were approved by the Committee of Laboratory Animals of Konkuk University (IACUC No. KU16187).

\section{Analysis of mouse liver function}

Serum levels of alanine aminotransferase (ALT) and aspartate aminotransferase (AST) were determined by measuring enzyme activities with a commercial assay kit using a Mindray BS400 automated spectrophotometer according to the manufacturer's instructions (Mindray Medical International Ltd., Shenzhen, China).

\section{Collagen staining in the mouse liver tissues}

Mouse liver tissues were fixed with $10 \%$ neutral formalin and embedded in paraffin. The paraffin sections were stained with hematoxylin and eosin (H\&E). Histological visualization of collagenous connective tissues was stained with a Trichrome Staining kit (ab150686 Connective Tissue stain; Abcam, Cambridge, UK) and collagen I and III fibers were stained with a Picro Sirius Red Staining Kit (ab150681 Connective Tissue stain; Abcam) according to the manufacturer's instructions.

\section{Cell line and cell culture}

HSCs were purchased from the American Type Culture Collection (ATCC, Manassas, VA, USA) and cultured in DMEM containing $10 \%$ fetal bovine serum (Invitrogen Life Technologies, Carlsbad, CA, USA) and antibioticanti-mycotic solution (Invitrogen Life Technologies) at $37{ }^{\circ} \mathrm{C}$ in a $5 \% \mathrm{CO}_{2}$ incubator.
Fig. 1 Inhibitory effect of Schisandra berry ethanolic extract (SBE) on TAA-induced liver damage. (A) Schematic illustration of the experimental design. Mice were treated with PBS, $100 \mathrm{mg} / \mathrm{kg}$ TAA alone or TAA with $150 \mathrm{mg} / \mathrm{kg}$ SBE. (B, C) Effect of TAA alone or TAA with SBE on the serum levels of $\operatorname{ALT}(\mathbf{B})$ and AST (C). Data are presented as the mean \pm SD $(n=6)$. The $P$ value was analyzed using ANOVA followed by Sidak's multiple comparisons test
(A)
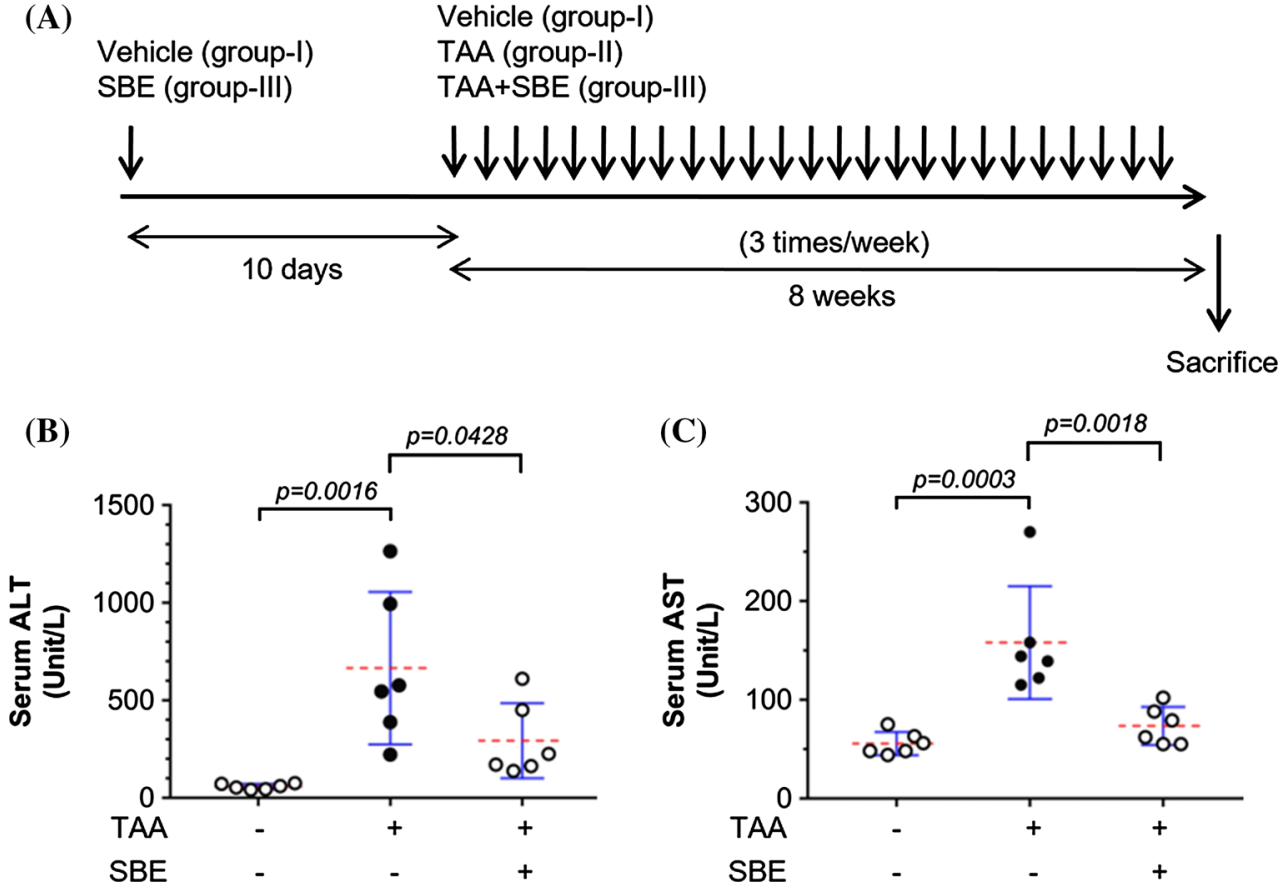
Fig. 2 Effect of Schisandra berry ethanolic extract (SBE) on TAA-induced collagen deposition in mouse liver. Liver specimens were fixed in $10 \%$ formalin and stained with $\mathrm{H} \& \mathrm{E}$ (A cytoplasm, pink; nucleus, purple), modified Masson's trichrome (B collagen, blue), and Picro Sirius Red

(C collagen fibers, red)

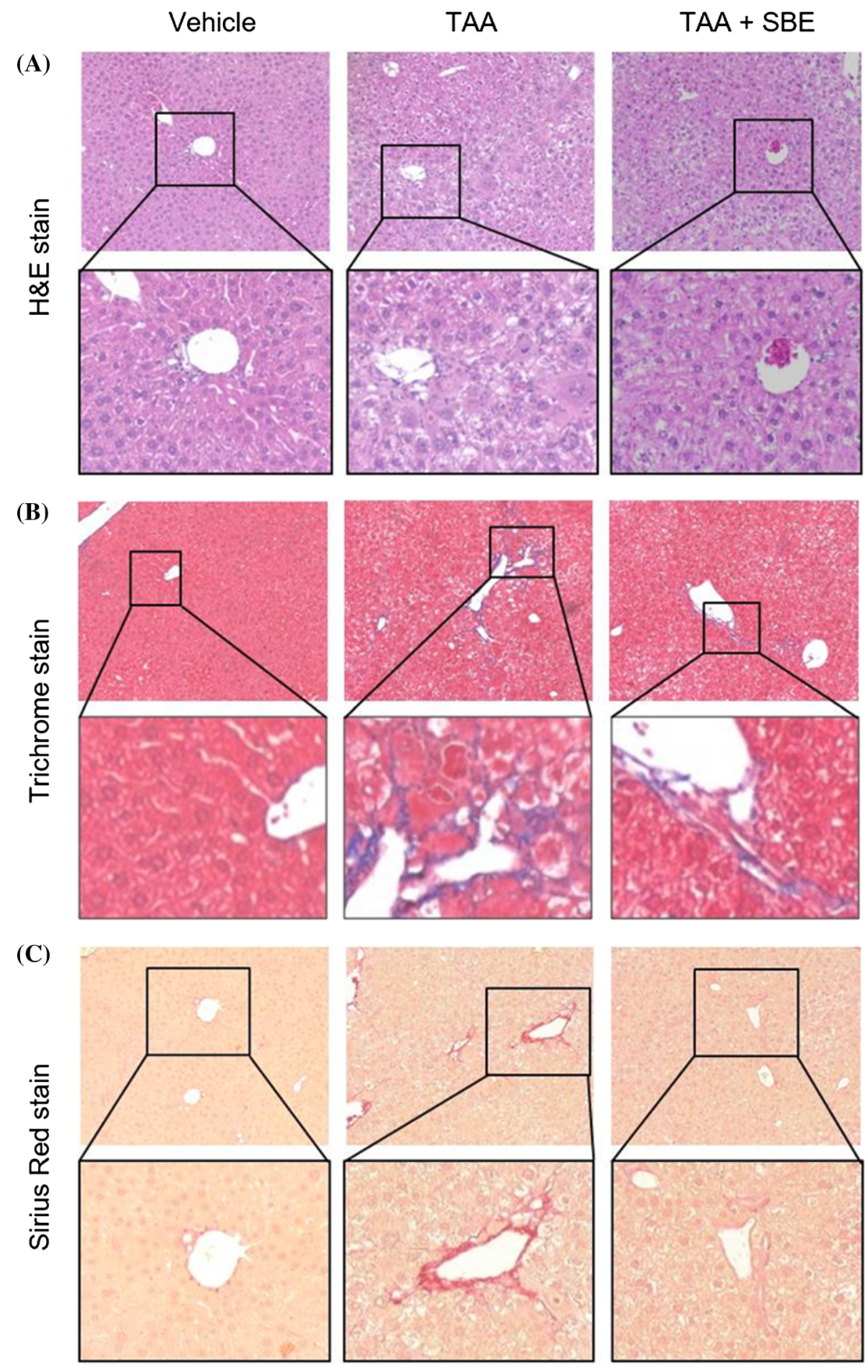

TAA

\section{Reverse transcription-polymerase chain reaction (RT-PCR)}

Total RNAs were isolated using Isol-RNA lysis reagent (NucleoZOL; Clontech, Mountain View, CA, USA). Double-stranded cDNAs were synthesized using an iScript cDNA synthesis kit (Bio-Rad, Hercules, CA, USA), according to the manufacturer's instructions. The primer sequences were synthesized by Macrogen (Seoul, Korea) as follows: actin forward, $5^{\prime}$-TGGAATCCTGTGGCATC CATGAAAC-3'; actin reverse, 5'-TAAAACGCAGCT CAGTAACAGTCCG-3'; $\alpha$ SMA forward, 5'-CTGAGCGT GGCTATTCCTT-3'; $\alpha$ SMA reverse, 5'-CTTCTGCATCC TGTCAGCAA-3'; COL1A1 forward, 5'-GACGCCATCA AGGTCTACTG-3'; COL1A1 reverse, 5'-ACGGGAATCC ATCGGTCA-3'. 
(A)

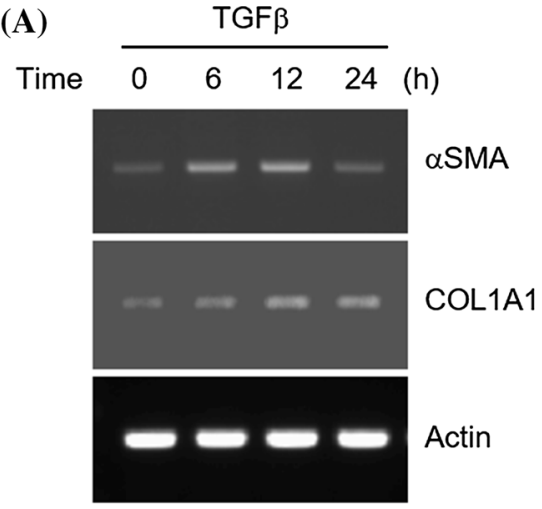

(C)

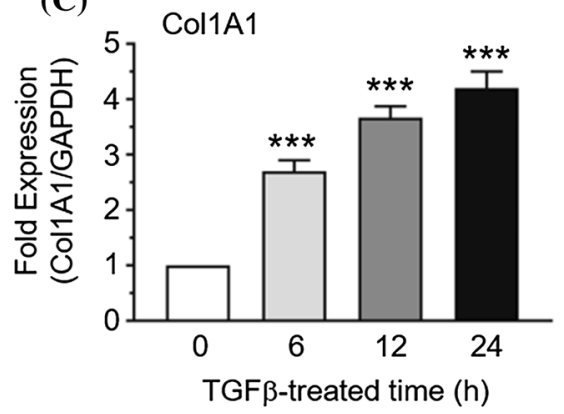

Fig. 3 Effect of TGF $\beta$ on the expression of $\alpha$ SMA and COL1A1 mRNAs. HSCs were treated with $10 \mathrm{ng} / \mathrm{mL}$ TGF $\beta$ for different time periods. (A) Total RNA was isolated, and aSMA and COL1A1 mRNA expression was measured by RT-PCR. Actin mRNA level was used as an internal control. (B, C) Total RNA was isolated, and aSMA (B) and COL1A1 (C) mRNA expression was measured by Q-PCR. Relative fold changes were normalized to those of GAPDH

\section{Quantitative real time-PCR (Q-PCR)}

The relative expression levels of $\alpha \mathrm{SMA}$ and GAPDH mRNAs were measured with a TaqMan-iQ ${ }^{\mathrm{TM}}$ supermix kit (Bio-Rad) using the Bio-Rad iCycler $\mathrm{iQ}^{\mathrm{TM}}$ according to the manufacturer's instructions. TaqMan ${ }^{\mathrm{TM}}$ fluorogenic probes and PCR probes were synthesized by Metabion International (Martinsried, Germany) as follows: COL1A1 TaqMan probe, 5'-FAM-CATCGTGGCTTCTCTGGTCT CC-BHQ-1-3'; aSMA TaqMan probe, 5'-FAM- TAGAAC ACGGCATCATCACCAACTG-BHQ-1-3'; glyceraldehyde-3-phosphate dehydrogenase (GAPDH) Taqman probe, 5'-YY-CGTCGCCAGCCGAGCCACATCGCBHQ-1-3' (FAM, 6-carboxyfluorescein; BHQ, tert-butylhydroquinone; YY, Yakima Yellow). Expression values were normalized to that of GAPDH mRNA in the same samples using the software program provided by the manufacturer.

\section{Statistical analysis}

Statistical significance was determined by one-way analysis of variance followed by Sidak's multiple comparisons
(B)

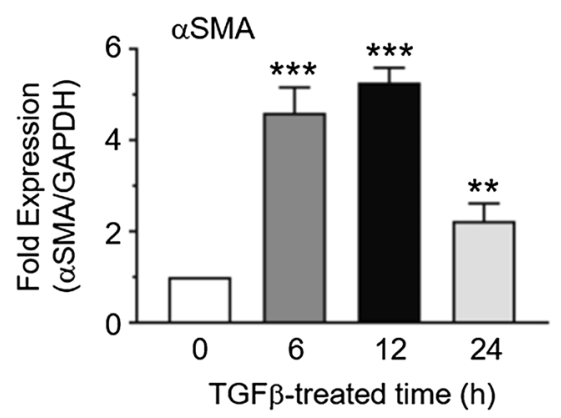

(D)

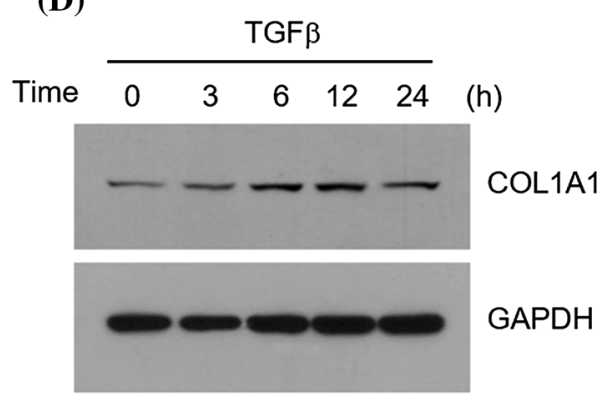

mRNA. Data are presented as the mean $\pm \mathrm{SD}(n=3) . * * P=0.009$; *** $P<0.001$ by ANOVA followed by Dunnett's multiple comparisons test. (D) HSCs were treated with $10 \mathrm{ng} / \mathrm{mL}$ TGF $\beta$ for different time periods. Whole cell lysates were prepared, and immunoblot analysis was performed with antibody against COL1A1. GAPDH level was measured as an internal control

test using GraphPad Prism version 7.02 (GraphPad Software, Inc., La Jolla, CA, USA).

\section{Results and discussion}

\section{Effect of Schisandra berry ethanolic extract (SBE) on TAA-induced liver injury}

When liver cells are damaged, liver ALT and AST enzymes are released into the blood. Therefore, increases in plasma ALT and AST levels are commonly used as a key biochemical marker for hepatotoxicity. Thioacetamide (TAA) is a hepatotoxin that causes acute hepatic failure in exposed animals [13]. To test the potential of SBE in protecting against liver injury, mice were divided into three groups and treated with vehicle, TAA, or TAA in combination with SBE for 8 weeks: G1, vehicle only; G2, TAA alone; and G3, SBE for 10 days, followed by SBE plus TAA (Fig. 1A). Treatment with TAA alone (3 times/week for 8 weeks; G2 group) significantly elevated the serum levels of ALT (Fig. 1B) and AST (Fig. 1C) compared to those in the control G1 group, indicating that TAA causes 

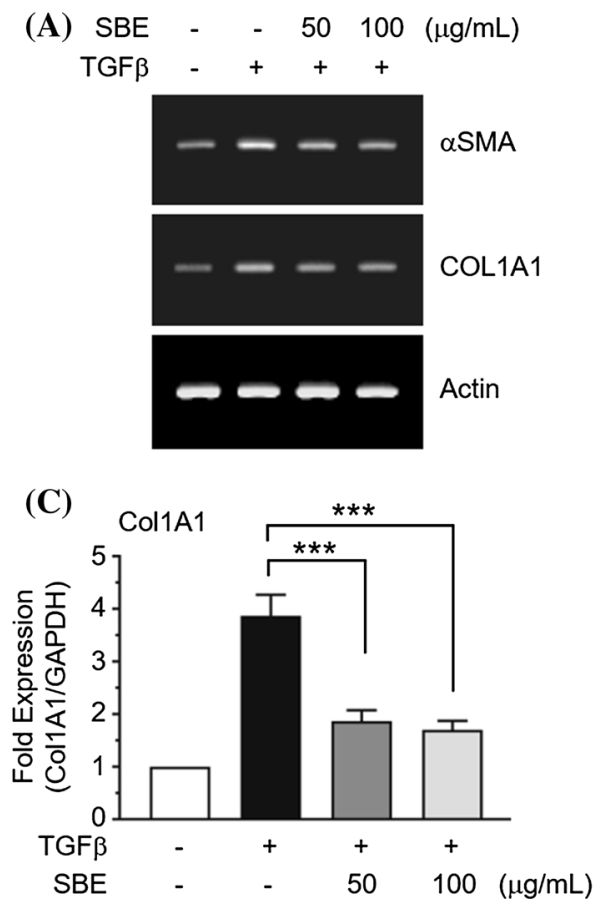

Fig. 4 Inhibitory effect of Schisandra berry ethanolic extract (SBE) on TGF $\beta$-induced expression of $\alpha \mathrm{SMA}$ and COL1A1 mRNAs. (A) HSCs were either untreated or treated with 50 or $100 \mu \mathrm{g} / \mathrm{mL} \mathrm{SBE}$ for $30 \mathrm{~min}$, followed by stimulation with $10 \mathrm{ng} / \mathrm{mL}$ TGF $\beta$. After $12 \mathrm{~h}$, total RNA was isolated and $\alpha \mathrm{SMA}$ and COL1A1 mRNA expression was measured by RT-PCR. Actin mRNA level was used as an internal control. (B, C) HSCs were treated as in (A), and total RNA was isolated. aSMA (B) and COL1A1 (C) mRNA expression was

hepatotoxicity under our experimental conditions. However, in the presence of SBE, TAA-induced ALT and AST activities were significantly (all $P<0.05$ by Sidak's multiple comparison test) reduced compared to those in the TAA-treated G2 group. These data suggest that SBE may have a beneficial effect against acute TAA-induced hepatotoxicity.

\section{SBE ameliorates TAA-induced hepatic fibrosis}

In TAA-treated livers, morphological features of parenchymal cells and lobular architectures were severely altered compared to those of the vehicle-treated control, whereas in the SBE-administered group, liver damage by TAA remarkably improved (Fig. 2A). It has been demonstrated that TAA-induced liver injury causes hepatic fibrosis [14]. The most characteristic feature of hepatic fibrosis is excessive deposition of extracellular matrix proteins, particularly collagen fibers. To determine the effect of SBE on the protection of hepatic fibrosis, collagen accumulation was visualized by modified Masson's trichrome staining (blue staining; Fig. 2B) and Picro Sirius Red staining (red staining; Fig. 2C). In TAA-treated hepatic tissue, severe deposition of collagen was observed.

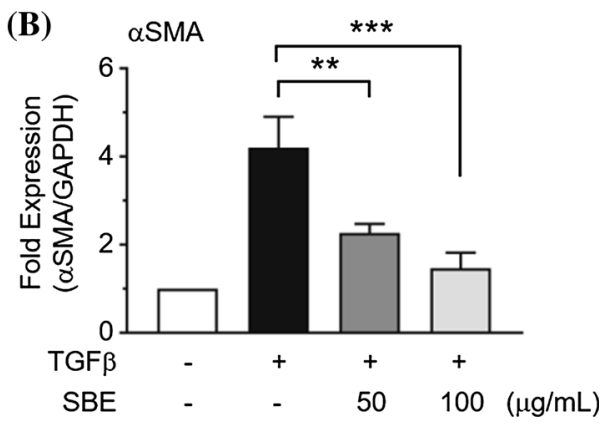

(D)

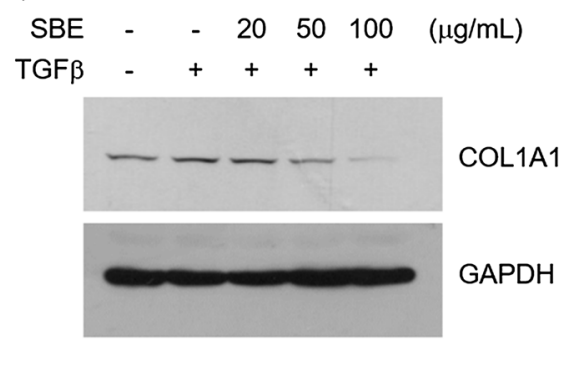

measured by Q-PCR. The relative fold changes were normalized to GAPDH mRNA. Data are presented as the mean $\pm \mathrm{SD}(\mathrm{n}=3)$. ** $P=0.002$; *** $P<0.001$ by ANOVA followed by Dunnett's multiple comparisons test. (D) HSCs were either untreated or treated with 20,50 or $100 \mu \mathrm{g} / \mathrm{mL}$ SBE for $30 \mathrm{~min}$, followed by stimulation with $10 \mathrm{ng} / \mathrm{mL}$ TGF $\beta$. After $12 \mathrm{~h}$, whole cell lysates were prepared and immunoblot analysis was performed with antibody against COL1A1. GAPDH level was measured as an internal control

However, TAA-induced collagen fibers were substantially decreased in SBE-treated liver tissue. These data suggest that SBE inhibits the deposition of collagen fibers induced by TAA in the mouse liver.

\section{TGF $\beta$ increases $\alpha$ SMA and COL1A1 expression in HSCs}

HSCs play a crucial role in the production of collagens in response to liver injury [3]. HSCs are in the perisinusoidal space between sinusoid endothelial cells and hepatocytes. TGF $\beta$, which is secreted from injured hepatocytes and Kupffer cells, triggers activation of quiescent HSCs into myofibroblast-like cells [15]. Activated HSCs expressing aSMA enhance the synthesis of matrix proteins, including COL1A1, and promote hepatic fibrosis [16]. To assess the effect of TGF $\beta$ on HSC activation, we examined the expression of $\alpha \mathrm{SMA}$ and COL1A1 mRNAs by reverse transcription-polymerase chain reaction (RT-PCR). Following TGF $\beta$ stimulation, the $\alpha$ SMA mRNA level increased within $6 \mathrm{~h}$ and was sustained for at least $12 \mathrm{~h}$ and COL1A1 expression gradually increased for up to $24 \mathrm{~h}$ after treatment (Fig. 3A). Q-PCR showed that aSMA mRNA levels increased by $4.6 \pm 0.56-, 5.3 \pm 0.32-$, and 


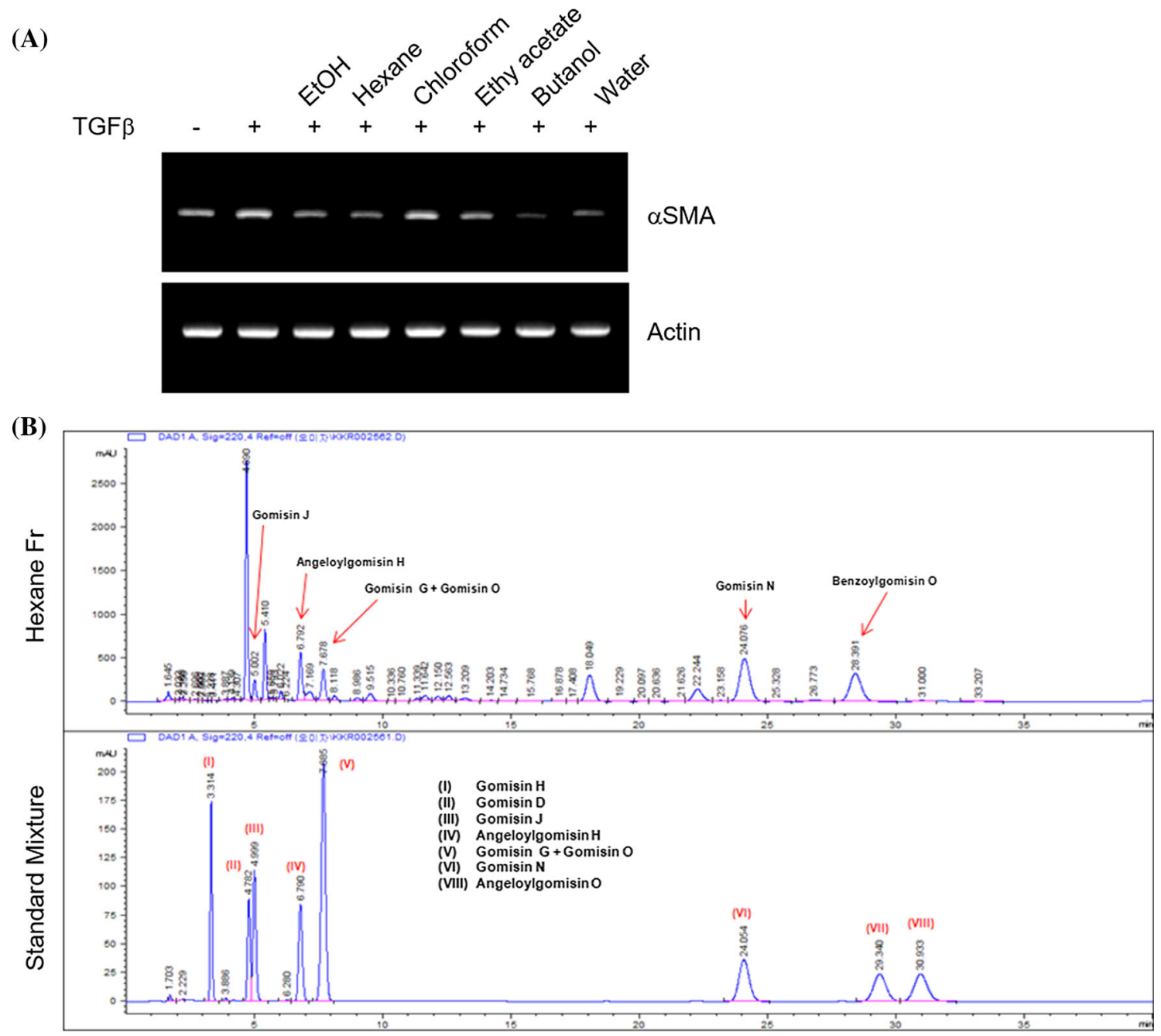

Fig. 5 Effect of sequential fractions of Schisandra berry on TGF $\beta$ induced expression of $\alpha$ SMA mRNA. (A) HSCs were either untreated or treated with $20 \mu \mathrm{g} / \mathrm{mL}$ fractions for $30 \mathrm{~min}$, followed by stimulation with $10 \mathrm{ng} / \mathrm{mL}$ TGF $\beta$. After $12 \mathrm{~h}$, total RNA was isolated and

$2.3 \pm 0.38$-fold $\quad$ (all $P<0.01) \quad$ (Fig. 3B), whereas COL1A1 mRNA levels were increased by $2.7 \pm 0.20$-, $3.67 \pm 0.21-, \quad$ and $4.2 \pm 0.30$-fold (all $P<0.001$ ) (Fig. 3C), respectively, after 6,12 , and $24 \mathrm{~h}$ of TGF $\beta$ stimulation. TGF $\beta$-induced elevation of COL1A1 protein levels was confirmed by immunoblotting (Fig. 3D). These data demonstrate that TGF $\beta$ stimulates HSCs in a timedependent manner under our experimental conditions.

\section{SBE inhibits TGF $\beta$-induced activation of hepatic stellate cells}

We next investigated whether SBE can inhibit HSCs stimulated by TGF $\beta$. HSCs were pre-treated with 50 and $100 \mu \mathrm{g} / \mathrm{mL}$ SBE before the addition of TGF $\beta$. RT-PCR showed that TGF $\beta$-induced $\alpha \mathrm{SMA}$ and COL1A1 expression was considerably attenuated in the presence of SBE
aSMA mRNA expression was measured by RT-PCR. Actin mRNA level was used as an internal control. (B) HPLC chromatogram of the $n$-hexane fraction (top) and chromatogram of the authentic lignans (bottom)

(Fig. 4A). To precisely quantify the inhibitory activity of SBE on TGF $\beta$-induced HSC activation, Q-PCR analysis was performed. Expression levels of $\alpha$ SMA (Fig. 4B) and COL1A1 (Fig. 4C) mRNAs were increased by $4.2 \pm 0.70$ and $3.5 \pm 0.35$-fold (all $P<0.001$ ), respectively, compared to those of the control group by TGF $\beta$ stimulation. SBE treatment attenuated these TGF $\beta$ inducibilities of both aSMA and COL1A1 mRNAs in a dose-dependent manner. Inhibitory effect of SBE on TGF $\beta$-induced COL1A1 expression was confirmed by immunoblotting (Fig. 4D). These results suggest that SBE inhibits TGF $\beta$-induced HSC activation. 


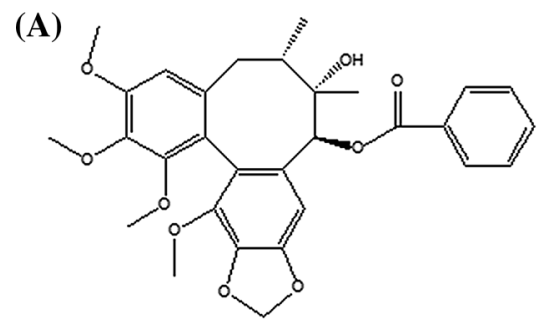

gomisin $G$<smiles>COC1=C(OC)C(O)C(O)CC2=C1c1c(cc(O)c(OC)c1OC)C[C@H](C)C(C)C2</smiles>

gomisin $\mathrm{J}$<smiles>COc1cc2c(c(OC)c1OC)-c1c(cc3c(c1OC)OCO3)C[C@@H](C)C(C)[C@H](O)[C@H]2O</smiles>

gomisin $\mathrm{O}$<smiles>C/C=C(/C)C(=O)Oc1c(OC)c(OC)cc2c1-c1c(cc(OC)c(OC)c1OC)C[C@@H](C)[C@](C)(O)C[C@@H]2C</smiles>

angeloylgomisin $\mathrm{H}$

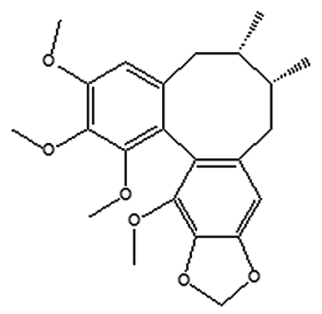

gomisin $\mathrm{N}$

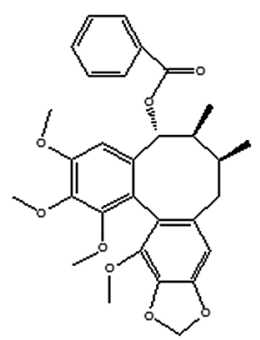

benzoylgomisin $\mathrm{O}$

(B)
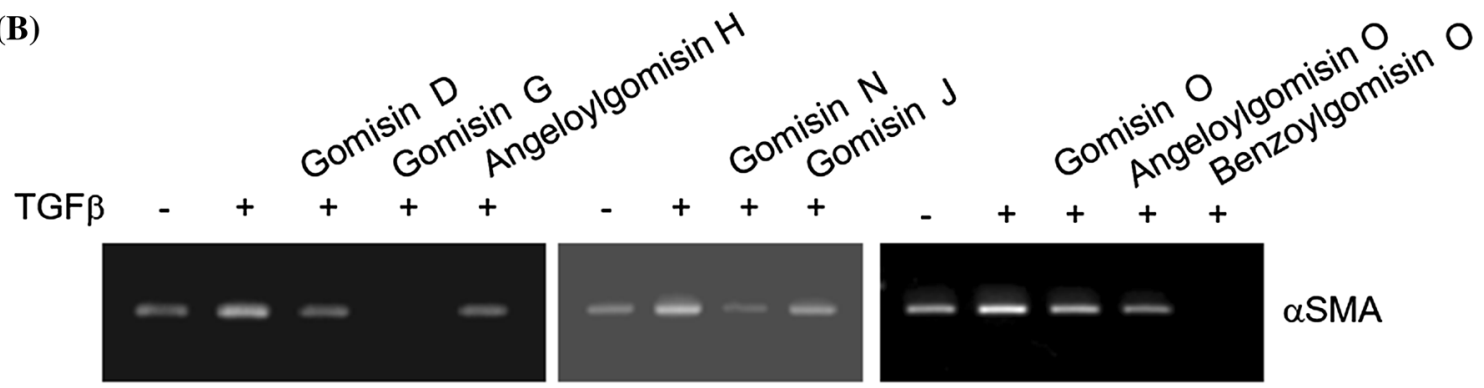

aSMA
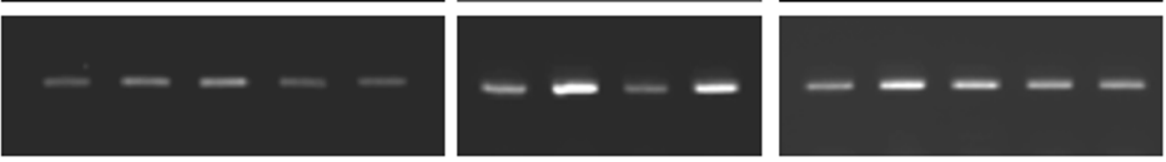

COL1A1
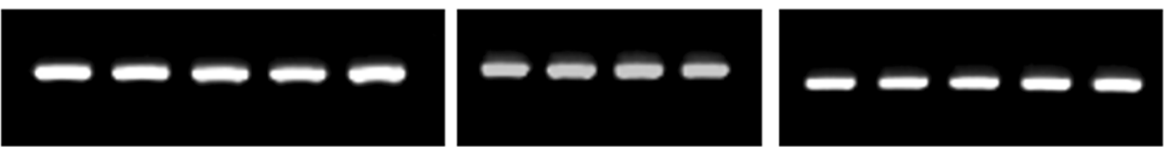

Actin

Fig. 6 Inhibitory effect of lignans contained in Schisandra chinensis on TGF $\beta$-induced expression of $\alpha$ SMA and COL1A1 mRNAs.

(A) The structures of six lignans identified in the $n$-hexane fraction.

(B) HSCs were either untreated or treated with $100 \mu \mathrm{g} / \mathrm{mL}$ solvent

\section{Identification of functional components of SBE responsible for suppression of TGFß-induced activation of HSCs}

To identify the functional components of SBE that inhibit HSCs, SBE was further partitioned successively with $n$ hexane, chloroform, ethyl acetate, $n$-butanol, and water. HSCs were treated with each fraction before TGF $\beta$ stimulation, and then aSMA mRNA levels were measured by RT-PCR. We found that the $n$-hexane and $n$-butanol fractions of SBE had strong inhibitory effects on TGF $\beta$-induced aSMA expression (Fig. 5A). We chose the $n$-hexane extracts for $30 \mathrm{~min}$, followed by stimulation with $10 \mathrm{ng} / \mathrm{mL}$ TGF $\beta$. After $12 \mathrm{~h}$, total RNA was isolated and $\alpha \mathrm{SMA}$ and COL1A1 mRNA expression was measured by RT-PCR. Actin mRNA level was used as an internal control

fraction because more components were observed in the chromatogram of this fraction compared to those in the $n$ butanol fraction. Because many lignans have been reported to be contained in S. chinensis as mentioned above, authentic samples of nine lignans including gomisin $\mathrm{D}$, gomisin $\mathrm{G}$, gomisin $\mathrm{H}$, gomisin $\mathrm{J}$, gomisin $\mathrm{N}$, gomisin $\mathrm{O}$, angeloylgomisin $\mathrm{H}$, angeloylgomisin $\mathrm{O}$, and benzoylgomisin $\mathrm{O}$ were prepared at a concentration of $1 \mathrm{~g} / \mathrm{L}$ in methanol. For HPLC analysis, $700-\mu \mathrm{L}$ samples were collected and mixed. The chromatogram obtained is shown in Fig. 5B bottom. Six lignans including gomisin G, gomisin $\mathrm{J}$, gomisin $\mathrm{N}$, gomisin $\mathrm{O}$, angeloylgomisin $\mathrm{H}$, and 
benzoylgomisin $\mathrm{O}$ were observed in the chromatogram of the $n$-hexane fraction (Fig. 5B, top). Although many studies have reported the hepatoprotective effects of lignan compounds isolated from $S$. chinensis, little information is available regarding the inhibitory effect on HSCs.

To determine whether lignan components exhibit inhibitory activity on TGF $\beta$-stimulated HSCs, we used commercially available lignans (Fig. 6A). HSCs were treated with different lignans before stimulation with TGF $\beta$, and aSMA and COL1A1 mRNA levels were measured. RTPCR revealed that six lignans (gomicin $\mathrm{G}$, gomicin $\mathrm{O}$, gomisin $\mathrm{J}$, gomicin $\mathrm{N}$, angeloylgomisin $\mathrm{H}$, and benzoylgomisin O) reduced TGF $\beta$-induced expression of both aSMA and COL1A1 mRNA (Fig. 6B). These data suggest that at least six lignans in SBE have the potential to prevent TGF $\beta$-induced activation of HSCs.

HSCs are primary cells that produce type-I collagen, a predominant extracellular matrix protein in liver fibrosis [17]. TGF $\beta$ is the most potent factor in promoting liver fibrosis by expressing extracellular matrix proteins $[16,18]$. During hepatic injury, TGF $\beta$ is secreted from hepatocytes, platelets, Kupffer cells, and sinusoidal endothelial cells [19]. It activates HSCs and then stimulates Smad2 and Smad3 transcription factors that regulate transcription of various target-genes including COL1A1 [20]. Type-I collagen is composed of the $\alpha 1$ and $\alpha 2$ chains encoded by the COL1A 1 and COL1A2 genes, respectively [21]. The previous study has reported that overexpression of Smad3 is sufficient to express COL1A1, suggesting that Smad transcription factors are essential in TGF $\beta$-induced COL1A1 expression.

Previously, Park et al. [22] have demonstrated that p38 and JNK mitogen-activated protein kinase (MAPK) pathways stimulate $\mathrm{Smad} 2 / 3$ and gomicin $\mathrm{N}$ (also called schisandrin B) protects TGF $\beta$-induced vascular fibrosis through inhibition of p38 and JNK MAPKs in rat aortic smooth muscle cell. Also, Chen et al. [12] have reported that gomicin $\mathrm{N}$ protects carbon tetrachloride $\left(\mathrm{CCL}_{4}\right)$-induced liver fibrosis by inhibition of TGF $\beta$-mediated Smad signaling pathway. Similarly, it is possible that SBE inhibits TGF $\beta$-induced COL1A1 expression through inhibition of Smad signaling pathway. Oxidative stress is closely associated with liver fibrosis [23]. Indeed, TGF $\beta$-induced oxidative stress up-regulates COL1A1 gene expression via the activation of a CCAAT/enhancer binding protein- $\beta(\mathrm{C} /$ EBP $\beta$ ) [24]. It has been reported that gomisin $A$ and gomicin $\mathrm{N}$ inhibit ROS production in lipopolysaccharidetreated microglial cells [25] and doxorubicin-treated mouse heart [26]. Thus, it is also possible that lignans in SBE may abrogate TGF $\beta$-induced COL1A1 expression through reducing of ROS accumulation in HSCs. Whether each lignan targets the same signaling pathway warrants further investigation. Future studies should explore which signaling pathway is responsible for mediating the inhibitory effect of each lignan on COL1A1 expression in HSCs.

In summary, this study showed that SBE inhibits TGF $\beta$ induced $\alpha$ SMA and COL1A1 mRNA expression in HSCs. We also found that at least six lignans (gomicin $\mathrm{G}$, gomicin $\mathrm{O}$, gomicin $\mathrm{N}$, gomisin $\mathrm{J}$, angeloylgomisin $\mathrm{H}$, and benzoylgomisin $\mathrm{O}$ ) in the $n$-hexane fraction of the SBE were functionally involved in inhibiting TGF $\beta$-induced HSC activation. Given that activation of HSCs plays a critical role in hepatic fibrogenesis in the injured liver, SBE can be utilized as a functional ingredient for preventing hepatic fibrosis.

Acknowledgment This study was supported by the Agri-Bio Industry Technology Development Program (316028-3, Korea Institute of Planning and Evaluation for Technology in Food, Agriculture, Forestry and Fisheries (IPET). SY Shin and J Lee contributed equally to this work. This paper was supported by the KU Research Professor Program of Konkuk University.

\section{References}

1. Bataller R, Brenner DA (2005) Liver fibrosis. J Clin Investig 115:209-218

2. Geerts A (2001) History, heterogeneity, developmental biology, and functions of quiescent hepatic stellate cells. Semin Liver Dis 21:311-335

3. Wu J, Zern MA (2000) Hepatic stellate cells: a target for the treatment of liver fibrosis. J Gastroenterol 35:665-672

4. Gressner AM, Bachem MG (1990) Cellular sources of noncollagenous matrix proteins: role of fat-storing cells in fibrogenesis. Semin Liver Dis 10:30-46

5. Okazaki I, Watanabe T, Hozawa S, Arai M, Maruyama K (2000) Molecular mechanism of the reversibility of hepatic fibrosis: with special reference to the role of matrix metalloproteinases. J Gastroenterol Hepatol 15(Suppl):D26-D32

6. Hancke JL, Burgos RA, Ahumada F (1999) Schisandra chinensis (Turcz.) Baill. Fitoterapia 70:451-471

7. Szopa A, Ekiert R, Ekiert H (2017) Current knowledge of Schisandra chinensis (Turcz.) Baill. (Chinese magnolia vine) as a medicinal plant species: a review on the bioactive components, pharmacological properties, analytical and biotechnological studies. Phytochem Rev 16:195-218

8. Hwang IS, Kim JE, Lee YJ, Kwak MH, Choi YH, Kang BC, Hong JT, Hwang DY (2013) Protective effects of gomisin A isolated from Schisandra chinensis against $\mathrm{CCl}(4)$-induced hepatic and renal injury. Int J Mol Med 31:888-898

9. Ip SP, Poon MK, Che CT, Ng KH, Kong YC, Ko KM (1996) Schisandrin $\mathrm{B}$ protects against carbon tetrachloride toxicity by enhancing the mitochondrial glutathione redox status in mouse liver. Free Radic Biol Med 21:709-712

10. Yamada S, Murawaki Y, Kawasaki H (1993) Preventive effect of gomisin A, a lignan component of shizandra fruits, on acetaminophen-induced hepatotoxicity in rats. Biochem Pharmacol 46:1081-1085

11. Kim M, Lim SJ, Lee HJ, Kim SY, Nho CW (2015) Gomisin J inhibits oleic acid-induced hepatic lipogenesis by activation of the AMPK-dependent pathway and inhibition of the hepatokine fetuin-A in HepG2 cells. J Agric Food Chem 63:9729-9739

12. Chen Q, Zhang H, Cao Y, Li Y, Sun S, Zhang J, Zhang G (2017) Schisandrin B attenuates CCl4-induced liver fibrosis in rats by 
regulation of Nrf2-ARE and TGF-beta/Smad signaling pathways. Drug Des Dev Ther 11:2179-2191

13. Chen TM, Subeq YM, Lee RP, Chiou TW, Hsu BG (2008) Single dose intravenous thioacetamide administration as a model of acute liver damage in rats. Int J Exp Pathol 89:223-231

14. Weiler-Normann C, Herkel J, Lohse AW (2007) Mouse models of liver fibrosis. Z Gastroenterol 45:43-50

15. Qi Z, Atsuchi N, Ooshima A, Takeshita A, Ueno H (1999) Blockade of type beta transforming growth factor signaling prevents liver fibrosis and dysfunction in the rat. Proc Natl Acad Sci U S A 96:2345-2349

16. Gressner AM, Weiskirchen R, Breitkopf K, Dooley S (2002) Roles of TGF-beta in hepatic fibrosis. Front Biosci 7:d793-d807

17. Pinzani M (1995) Novel insights into the biology and physiology of the Ito cell. Pharmacol Ther 66:387-412

18. Bedossa P, Paradis V (1995) Transforming growth factor-beta (TGF-beta): a key-role in liver fibrogenesis. J Hepatol 22:37-42

19. Gressner AM, Weiskirchen R (2006) Modern pathogenetic concepts of liver fibrosis suggest stellate cells and TGF-beta as major players and therapeutic targets. J Cell Mol Med 10:76-99

20. Schmierer B, Hill CS (2007) TGFbeta-SMAD signal transduction: molecular specificity and functional flexibility. Nat Rev Mol Cell Biol 8:970-982

21. Ramirez F, Di Liberto M (1990) Complex and diversified regulatory programs control the expression of vertebrate collagen genes. FASEB J 4:1616-1623
22. Park EJ, Chun JN, Kim SH, Kim CY, Lee HJ, Kim HK, Park JK, Lee SW, So I, Jeon JH (2012) Schisandrin B suppresses TGFbeta1 signaling by inhibiting Smad2/3 and MAPK pathways. Biochem Pharmacol 83:378-384

23. Kaplowitz N, Tsukamoto H (1996) Oxidative stress and liver disease. Prog Liver Dis 14:131-159

24. Garcia-Trevijano ER, Iraburu MJ, Fontana L, Dominguez-Rosales JA, Auster A, Covarrubias-Pinedo A, Rojkind M (1999) Transforming growth factor betal induces the expression of alpha1(I) procollagen mRNA by a hydrogen peroxide-C/EBPbeta-dependent mechanism in rat hepatic stellate cells. Hepatology 29:960-970

25. Wang X, Hu D, Zhang L, Lian G, Zhao S, Wang C, Yin J, Wu C, Yang J (2014) Gomisin A inhibits lipopolysaccharide-induced inflammatory responses in $\mathrm{N} 9$ microglia via blocking the NFkappaB/MAPKs pathway. Food Chem Toxicol 63:119-127

26. Thandavarayan RA, Giridharan VV, Arumugam S, Suzuki K, Ko KM, Krishnamurthy P, Watanabe K, Konishi T (2015) Schisandrin $\mathrm{B}$ prevents doxorubicin induced cardiac dysfunction by modulation of DNA damage, oxidative stress and inflammation through inhibition of MAPK/p53 signaling. PLoS ONE 10:e0119214 